\title{
Access to Highly Efficient Energy Transfer in Metal-Organic Frameworks via Mixed Linkers Approach
}

\author{
Jiangtao Jia, aæ Luis Gutiérrez-Arzaluz, bæ Osama Shekhah,a Norah Alsadun,a,c Justyna Czaban- \\ Jóźwiak,a Sheng Zhou,a Osman M. Bakr,b Omar F. Mohammed,b,*and Mohamed Eddaoudia* \\ a King Abdullah University of Science and Technology (KAUST), Functional Materials Design, Discovery and \\ Development Research Group $\left(\mathrm{FMD}_{3}\right)$, Advanced Membranes and Porous Materials Center (AMPMC), Division of \\ Physical Sciences and Engineering (PSE), Thuwal 23955-6900, Saudi Arabia. \\ b King Abdullah University of Science and Technology (KAUST), Division of Physical Sciences and Engineering \\ (PSE), Thuwal 23955-6900, Saudi Arabia. \\ c Department of Chemistry, College of Science, King Faisal University (KFU), Alahsa 31982-40o, Saudi Arabia.
}

Supporting Information Placeholder

\begin{abstract}
Herein, we report a new light-harvesting mixedligand $\mathrm{Zr}(\mathrm{IV})$-based metal-organic framework (MOF), with underlying fcu topology, encompassing the $\left[\mathrm{Zr} 6\left(\mu_{3}-\mathrm{O}\right)_{4}\left(\mu_{3^{-}}\right.\right.$ $\mathrm{OH})_{4}\left(\mathrm{O}_{2} \mathrm{C}-\right)_{12}$ ] cluster and an equimolar mixture of thiadiazole-and imidazole-functionalized ligands. The successful integration of ligands with similar structural features but with notable chemical distinction afforded the attainment of a highly efficient energy transfer. Notably, the very strong spectral overlap between the emission spectrum of benzimidazole (energy donor) and the absorption spectrum of thiadiazole (energy acceptor) provided an ideal platform to achieve very rapid (picosecond time scale) and highly efficient energy transfer (around 9o\% efficiency), as evidenced by timeresolved spectroscopy. Remarkably, the ultrafast timeresolved experiments quantified for the first time the anticipated close proximity of the two linkers with an average distance of $17 \AA$. This finding paves the way for the design and synthesis of periodic MOFs affording very efficient and fast energy transfer to mimic natural photosynthetic systems.
\end{abstract}

Photosynthesis is one of the key processes in nature, and it is principally based on an energy transfer process that takes place between chlorophyll (an antenna molecule) and a carotenoid (a pigment molecule) present in floras. The energy transfer process in plants occurs by means of the absorption of a wide range of wavelengths of sunlight that can be transferred to the reaction center via energy transfer and is then converted into chemical energy.1-3 Several attempts have been made to design highly efficient natural energy transfer systems, such as assemblies of covalently bonded porphyrin arrays, $_{4}$ dendrimers, 5 chromogenic polymers, 6 and selfassembled donor-acceptor supramolecular systems.7 This energy transfer process is most effective through assembly of an ordered network embedded within the same systems.8 Metal-organic frameworks (MOFs), an emerging class of porous crystalline materials, that are built up from multidentate organic building blocks and metal or metal clusters, 9 have paved their way as bulk and thin films in diverse applications, including gas separation and storage, 10 sensing, ${ }_{11}$ catalysis, 12 and drug release. 13 This versatility is a result of their permanent porosity, surface area, and structural and functional tunability. In particular, MOFs have been brought into the limelight as a suitable platform for exploration of directional energy transfer phenomena. This is mainly due to the distances and angles between linkers in MOF structures, which can be easily determined by different techniques such as single-crystal X-ray crystallography. The ability to design and fine-tune MOF structures can be approached methodically via the unlimited possibilities of altering and combining organic ligands and metals or metal clusters.14-15

In recent years, several research groups have made considerable progress in the design and development of MOFs for light-harvesting applications. In principle, energy transfer in MOFs can be introduced via various pathways, including metal-to-ligand, metal-to-metal, host-to-guest, and ligand-toligand scenarios.16-21 Several studies have reported stimulating strut-to-strut energy transfer pathways within MOFs encompassing porphyrin ligands.22-24 These early investigations encouraged us to explore novel structures containing two complementary ligands in a single MOF structure (mixed-ligand-based MOFs) to study ligand-toligand energy transfer. The mixed-ligand approach is the ideal design to co-assemble two ligands in a single MOF structure. Thus, we herein report a new zirconium-based mixed-ligand fcu MOF (Zr-ML-fcu-MOF), which was obtained by the solvothermal reaction between a zirconium salt and a equimolar mixture ratio of 4,4'-(1H-benzo[d]imidazole-4,7diyl)dibenzoic acid (BI) and 4,4'-(benzo[c][1,2,5]thiadiazole-

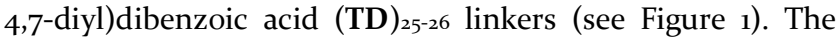
mixed-ligand strategy successfully yielded Zr-ML-fcu-MOF, because of the identical length, symmetry and connectivity of the two ligands (see Figure 1 ). The rapid and highly efficient ligand-to-ligand energy transfer from the benzimidazole linker to the thiadiazole linker in Zr-ML-fcu-MOF was confirmed by various techniques, such as steady-state and time-resolved luminescence measurements.

The new Zr-ML-fcu-MOF was synthesized by a conventional method, as described in Figure $1 . \mathrm{ZrCl}_{4}$ and TFA were mixed in DMF for $1 \mathrm{~h}$, followed by the addition of BI and TD solutions. Heating for $24 \mathrm{~h}$ at $120^{\circ} \mathrm{C}$ resulted in the formation of $\mathrm{Zr}-\mathrm{ML}$ fcu-MOF as pale yellow fine crystals. The synthetic procedures are described in detail in the experimental section in the supporting information (SI). For comparison purposes, we have also synthesized single-ligand MOFs (Zr-BI-fcu-MOF and Zr-TD-fcu-MOF) of BI and TD by a conventional method, which also yielded white and yellow fine crystal products, respectively. Figure 2 a shows the powder X-ray diffraction (PXRD) patterns of the three as-synthesized MOFs. The PXRD 


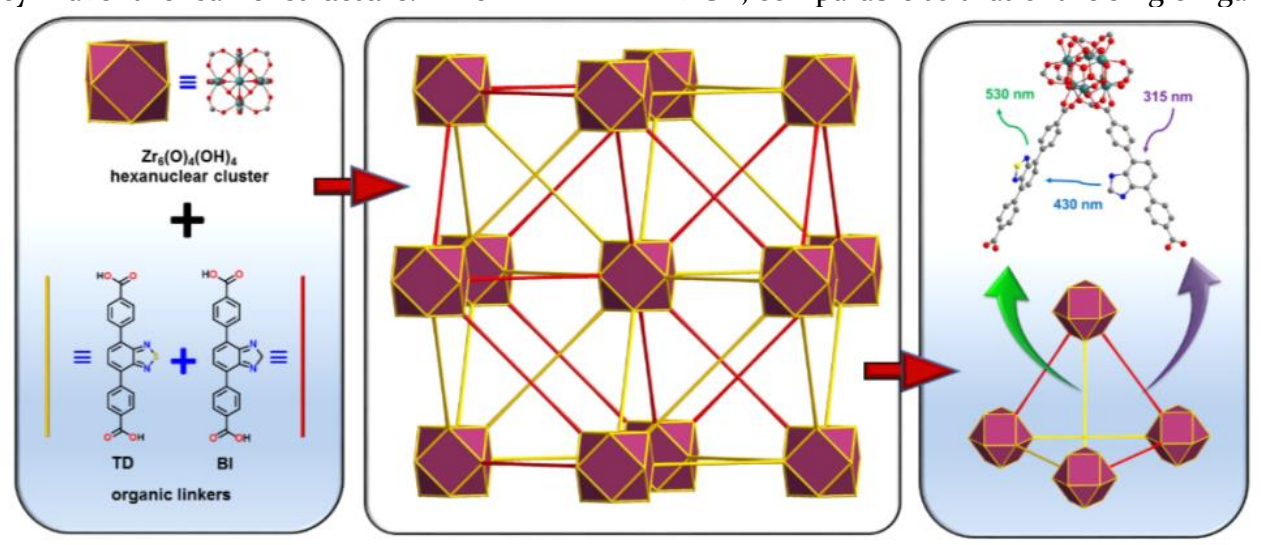

Figure 1. Schematic representation of the Zr-ML-fcu-MOF structure and its synthesis from the Zr precursor and the BI (represented as yellow line) and TD (represented as red line) mixed linkers. (inset) The chemical structure of the Zr-ML-fcuMOF in the tetrahedral cage with the different absorption and emission wavelengths of the two linkers.

${ }_{1} \mathrm{H}$ NMR measurements were conducted to determine the ratio of BI and TD linkers in these Zr-ML-fcu-MOFs. It should be noted that the ${ }_{1} \mathrm{H}$ NMR spectra were obtained after digestion of the Zr-ML-fcu-MOF in concentrated hydrochloric acid and separation of the organic ligands by centrifugation. ${ }_{1} \mathrm{H}$ NMR of the digested $\mathrm{Zr}-\mathrm{ML}-\mathrm{fcu}-\mathrm{MOF}$ shows signals from both ligands. As shown in Figure $1 \mathrm{~b}$, the peak at $8.08 \mathrm{ppm}$ could be attributed to the TD ligand, and the peak at $7.68 \mathrm{ppm}$ represents the proton from the BI ligand. By integrating the peaks from both ligands, we can conclude that the ratio between the BI and TD ligands is approximately 1:1.25. We also performed high-resolution X-ray photoelectron spectroscopy (XPS) (Figure $\mathrm{S}_{5}$ ) to determine the nitrogen $(\mathrm{N})$ and sulfur $(\mathrm{S})$ contents on the surface of Zr-ML-fcu-MOF. The results show that on the Zr-ML-fcu-MOF surface, both $\mathrm{N}$ and $\mathrm{S}$ are present, and by calculating the ratio of $\mathrm{N}$ to $\mathrm{S}$, we can conclude that the ratio of BI:TD ligands on the surface is approximately 1.1:1, which is very comparable to that determined from the ${ }_{1} \mathrm{H} \mathrm{NMR}$ data.

The scanning electron microscopy (SEM) image of the mixed ligand sample (Figure S6) shows that Zr-ML-fcu-MOF is mainly composed of one type of very small crystal particle. The elemental mapping scan of $\mathrm{N}$ and $\mathrm{S}$ for the Zr-ML-fcuMOF particles shows a uniform distribution of $\mathrm{N}$ and $\mathrm{S}$, which indicates that there is a good distribution of the two linkers in the MOF structure. From the N and S contents, we estimated the ratio of BI:TD ligands to be approximately 1:1.1 (Figure $\mathrm{S}_{7}$, and S8). The porosity of Zr-BI-fcu-MOF, Zr-TD-fcu-MOF, and $\mathrm{Zr}$-ML-fcu-MOF was evaluated via nitrogen adsorption-desorption isotherms at $77 \mathrm{~K}$ (Figures S9, S1o and Si1). All of Zr-BI-fcu-MOF, Zr-TD-fcu-MOF, and Zr-ML-fcuMOF have shown type I $\mathrm{N}_{2}$ isotherms. On the other hand, Brunauer-Emmett-Teller (BET) analysis of the $\mathrm{N}_{2}$ adsorption-desorption isotherms confirms that $\mathrm{Zr}$-BI-fcuMOF, Zr-TD-fcu-MOF, and Zr-ML-fcu-MOF have high surface areas of $\sim 3490,2380$ and $3110 \mathrm{~m}_{2} \mathrm{~g}_{-1}$ and pore volumes of $1.47,1.02$ and $1.26 \mathrm{~cm}_{3} \mathrm{~g}_{-1}$, respectively.

The efficient energy transfer in Zr-ML-fcu-MOF was visualized using steady-state absorption and photoluminescence (PL), and time-resolved spectroscopies. The steady-state spectra in Figure za show that $\mathrm{Zr}-\mathrm{BI}-\mathbf{f c u}-$ MOF has an absorption band at $350 \mathrm{~nm}$ and exhibits an emission spectrum between 400 and $550 \mathrm{~nm}$. Zr-TD-fcu-MOF (Figure 3b) shows a broad absorption spectrum at approximately $450 \mathrm{~nm}$ and an emission band at $530 \mathrm{~nm}$. As shown in Figure 3, the significant spectral overlap between the emission spectrum of $\mathrm{Zr}$-BI-fcu-MOF (donor) and the absorption spectrum of $\mathrm{Zr}$-TD-fcu-MOF (acceptor) strongly supports the energy transfer process from the $\mathbf{B I}$ linker to the TD linker.

Note that in addition to the distance between the two linkers, the degree of this spectral overlap is one of the key factors used to determine the rate and efficiency of Förster-type resonant energy transfer (FRET).27

We explore and decipher the interplay between the linkers in Zr-ML-fcu-MOF using steady-state absorption experiments, as shown in Figure 3c. Zr-ML-fcu-MOF has a broad absorption band from 350 to $500 \mathrm{~nm}$. This spectrum is consistent with contributions from the $\mathrm{Zr}$-BI-fcu-MOF band at $350 \mathrm{~nm}$ and that from $\mathrm{Zr}-\mathrm{TD}-\mathbf{f c u}-\mathrm{MOF}$ at approximately $450 \mathrm{~nm}$. 

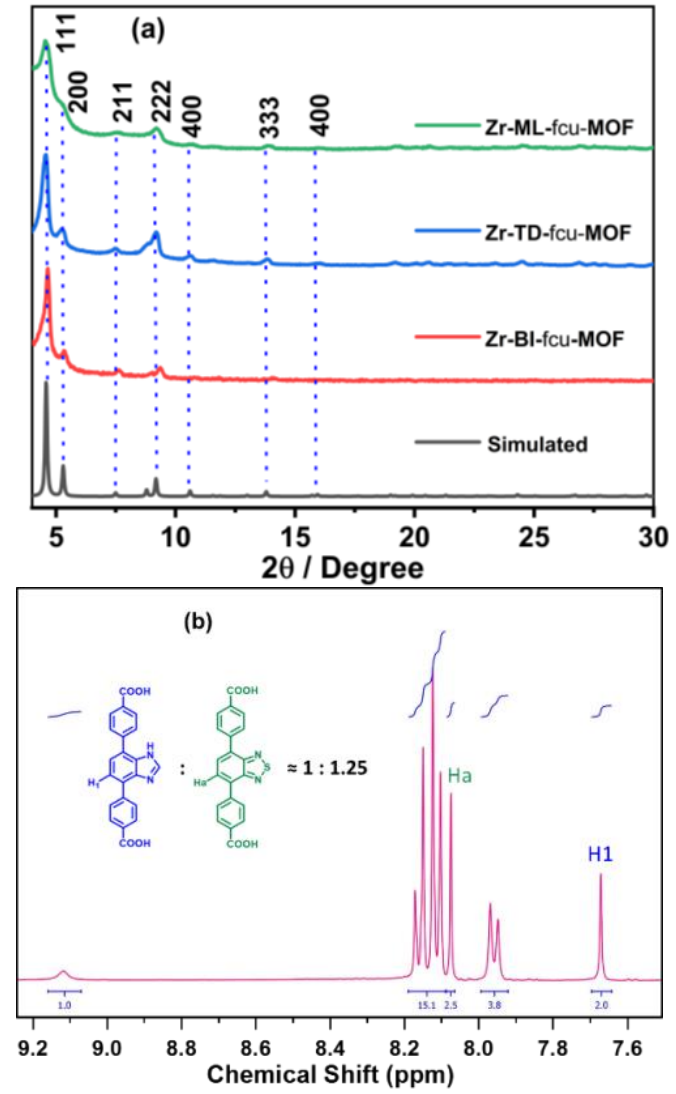

Figure 2. (a) PXRD patterns of the synthesized $\mathbf{Z r}-\mathbf{B I}-\mathrm{fcu}-$ MOF (red), Zr-TD-fcu-MOF (blue) and Zr-ML-fcu-MOF (green) and simulated PXRD pattern of UiO-68 (black); (b) ${ }_{1} \mathrm{H}$ NMR plot of the digested Zr-ML-fcu-MOF.

This observation also confirms the presence of both ligands in the MOF. On the other hand, from the emission spectra of $\mathrm{Zr}$ ML-fcu-MOF, we note that the disappearance of the BI emission peak upon $315 \mathrm{~nm}$ excitation provides an indication for energy transfer from BI to TD. The almost complete PL quenching of the $\mathbf{B I}$ emission band indicates that the process occurs in our Zr-ML-fcu-MOF with very high efficiency. This behavior is not observed in the physical mixture of free ligands and is not observed in the physically mixed Zr-BI-fcu-MOF and $\mathrm{Zr}$-TD-fcu-MOF (Figure S12-S14), highlighting the importance of the Zr-ML-fcu-MOF architecture that significantly facilitates the process due to the proximity and orientation of the ligands. In other words, the constrain produced by the MOF architecture, facilitates the energy transfer process and prevents other ligand excited-state deactivation channels to take place

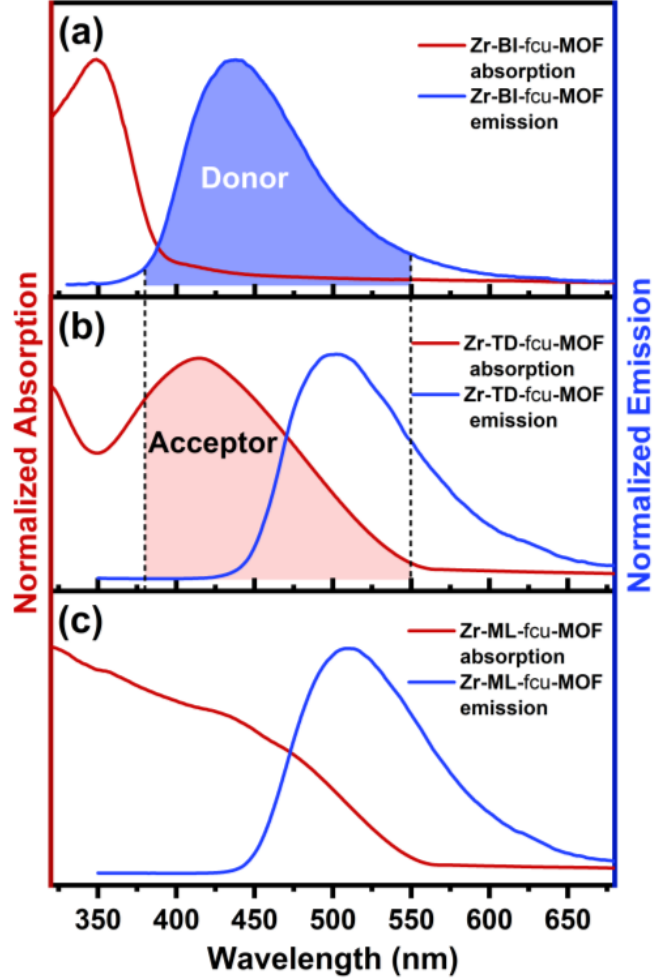

Figure 3. Absorption and emission spectra of $\mathbf{Z r}$-BI-fcu-MOF (a), Zr-TD-fcu-MOF (b), and Zr-ML-fcu-MOF (c) in acetonitrile. $\lambda_{\text {exc }}=315 \mathrm{~nm}$. The framed area shows the overlap between donor emission and acceptor absorption.

To confirm the energy transfer process and determine its characteristic rate, we performed time-resolved emission experiments through time-correlated single-photon counting technique (TCSPC). Namely, the BI band was monitored at $440 \mathrm{~nm}$, and the TD band was monitored at $530 \mathrm{~nm}$. The TCSPC data in Figure 4 show significant differences in the excited-state lifetimes of Zr-BI-fcu-MOF, Zr-TD-fcu-MOF, and $\mathrm{Zr}-\mathrm{ML}-\mathbf{f c u}-\mathrm{MOF}$. At early times (Figure 4a), the TD band in $\mathrm{Zr}-\mathrm{ML}-\mathbf{f c u}-\mathrm{MOF}$ ( $530 \mathrm{~nm}$ ) shows a PL rise compared with that of the respective single-ligand MOF. This accumulation could be attributed to the energy transfer from the BI to the TD. The extracted time constant of $108 \pm 12 \mathrm{ps}$ and the complete PL quenching of the donor suggest a rapid and highly efficient energy transfer between the two ligands. Complementing this evidence, the $440 \mathrm{~nm}$ emission band in Zr-ML-fcu-MOF exhibits an early fast decay compared to that of $\mathrm{Zr}-\mathrm{BI}-\mathbf{f c u}-\mathrm{MOF}$. It is worth mentioning that this energy transfer rate is faster compared to the ones found in other MOFs that exhibit nanosecond scale ligand-to-ligand energy transfer.14, 17, 21, 28

The exponential fittings for the kinetic traces on the long time scale in Figure $4 \mathrm{~b}$ show a diminution in the BI emission lifetime from $2.82 \pm 0.09 \mathrm{~ns}$ for $\mathrm{Zr}-\mathrm{BI}-\mathrm{fcu}-\mathrm{MOF}$ to $0.81 \pm 0.06$ ns for $\mathrm{Zr}-\mathrm{ML}-\mathbf{f c u}-\mathrm{MOF}$. This decrease is accompanied by an increase in the lifetime of the TD emission lifetime from $1.41 \pm$ $0.07 \mathrm{~ns}$ in Zr-TD-fcu-MOF to $3.17 \pm 0.06 \mathrm{~ns}$ in Zr-ML-fcuMOF. These changes also confirm the photoluminescence quenching of the BI by energy transfer to the TD. 

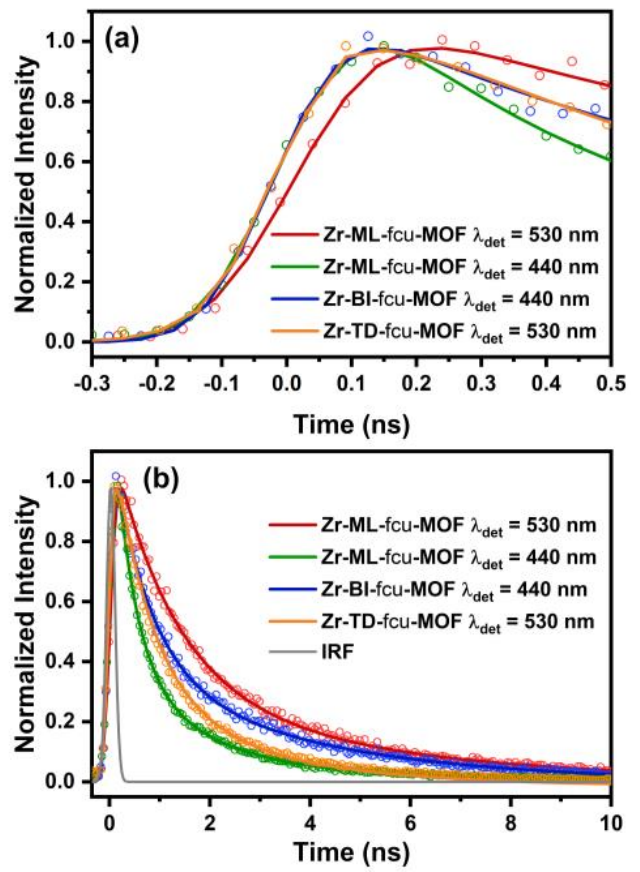

Figure 4. TCSPC data for Zr-BI-fcu-MOF, Zr-TD-fcu-MOF, and $\mathbf{Z r}-\mathbf{M L}$-fcu-MOF at their emission bands in acetonitrile solution (a) on an early time scale and (b) on a long time scale. The exponential fittings are indicated as solid lines. $\lambda_{\text {exc }}=350$ nm.

Using the lifetime and steady-state spectroscopic data, we first calculated the distance between the $\mathbf{B I}$ and TD ligands inside the $\mathrm{Zr}-\mathrm{ML}$-fcu-MOF through the Förster energy transfer equation (eq. 1).27

$$
k_{F R E T}=\frac{1}{\tau_{D}}\left(\frac{R_{0}}{r}\right)^{6}
$$

where KFRET is the rate of the energy transfer process obtained from PL lifetime measurements, $\tau D$ is the lifetime of the donor in the absence of the acceptor, $R_{\mathrm{o}}$ is the Förster distance, and $r$ is the distance between the donor and the acceptor units. More details about the values of the equation parameters are included in the S I. From the kinetic traces, the energy transfer time constant was determined to be 108 ps (the rise component in the TD kinetic traces in the $\mathrm{Zr}-\mathrm{ML}-\mathbf{f c u}-\mathrm{MOF}$ ). This time constant yielded a length of 17 Å between the two ligands. This value is in good agreement with the calculated values for neighboring ligands inside $\mathrm{Zr}$-fcu-MOFs. It should be noted that there are two types of pores in Zr-ML-fcu-MOF, octahedral and tetrahedral. The average distances between the two ligands in the tetrahedral and octahedral cages are approximately 12-17 $\AA$ and 12-24 $\AA$, respectively (Figure S15). This evidence also confirms that the ligands are evenly well distributed within the $\mathrm{Zr}-\mathrm{ML}$-fcu-MOF structure. The energy transfer most likely takes place between ligands at close proximity, where the probability of finding different ligands next to each other is high.

The time-resolved PL results also allow us to estimate the efficiency of energy transfer between the two linkers via eq. 2:29

$$
\epsilon=\frac{1}{1+\frac{1}{\tau_{D} k_{F R E T}}}
$$

The efficiency $(\epsilon)$ found in our system was $\sim 90 \%$. This high value is also consistent with the substantial decrease in the $\mathbf{B I}$ emission band in the steady-state experiments. Moreover, it confirms the capability of MOFs to serve as excellent lightharvesting materials in addition to all their known properties. It should be noted that the efficiency is higher than other imidazole derivatives MOFs ( $72 \%)_{28}$ and even porphyrinbased MOFs $(85 \%), 14,17,28$ establishing our MOF as a better energy-transfer system and a potential light-harvesting material template.

In summary, we have successfully synthesized a new $\mathrm{Zr}-\mathrm{ML}$ fcu-MOF based on mixed imidazole- and thiadiazolefunctionalized linkers. This new MOF structure exhibits very rapid and highly efficient energy transfer. More specifically, steady-state and time-resolved spectroscopic measurements demonstrate that $90 \%$ energy transfer efficiency with an approximately 100 ps time constant was achieved, representing one of the most efficient energy transfer processes in families of MOFs. This work highlights the key variable components to design and synthesize MOFs as new efficient light-harvesting materials. Moreover, since the interligand distance has a remarkable effect on the energy transfer process, the system could be slightly modified to tune its optical properties by controlling the ligand-ligand distance as have been proposed for other systems that undergo variable energy transfer process.3o

\section{ASSOCIATED CONTENT}

\section{Supporting Information}

The Supporting Information is available free of charge on the ACS Publications website. Synthesis, experimental data and procedures, X-ray diffraction, absorption and photoluminescence data.

\section{AUTHOR INFORMATION}

\section{Corresponding Author}

Mohamed

Eddaoudi:

mohamed.eddaoudi@kaust.edu.sa

Omar F. Mohammed: omar.abdelsaboor@kaust.edu.sa

\section{Author Contributions}

¥J.J. and L.G.A contributed equally.

\section{Notes}

The authors declare no competing financial interests.

\section{ACKNOWLEDGMENT}

The authors gratefully acknowledge financial support from King Abdullah University of Science and Technology (KAUST).

\section{REFERENCES}


(1) Barber, J.; Andersson, B., Revealing the Blueprint of Photosynthesis. Nature 1994, 370 (6484), 31-34.

(2) Cheng, Y. C.; Fleming, G. R., Dynamics of Light Harvesting in Photosynthesis. Annu. Rev. Phys. Chem. 2009, 6o, 241-262.

(3) Vangrondelle, R.; Dekker, J. P.; Gillbro, T.; Sundstrom, V., EnergyTransfer and Trapping in Photosynthesis. Biochim. Biophys. Acta, Bioenerg.. 1994, 1187 (1), 1-65.

(4) Aratani, N.; Kim, D.; Osuka, A., Discrete Cyclic Porphyrin Arrays as Artificial Light-Harvesting Antenna. Accounts. Chem. Res. 2009, 42 (12), 1922-1934.

(5) Astruc, D.; Boisselier, E.; Ornelas, C., Dendrimers Designed for Functions: From Physical, Photophysical, and Supramolecular Properties to Applications in Sensing, Catalysis, Molecular Electronics, Photonics, and Nanomedicine. Chem. Rev. 2010, 110 (4), 1857-1959.

(6) Webber, S. E., Photon-Harvesting Polymers. Chem. Rev. 1990, 90 (8), 1469-1482.

(7) Li, X. Y.; Sinks, L. E.; Rybtchinski, B.; Wasielewski, M. R., Ultrafast aggregate-to-aggregate energy transfer within self-assembled light-harvesting columns of zinc phthalocyanine tetrakis(perylenediimide). J. Am. Chem. Soc. 2004, 126 (35), 1081010811.

8. Wasielewski, M. R., Self-Assembly Strategies for Integrating Light Harvesting and Charge Separation in Artificial Photosynthetic Systems. Accounts. Chem. Res. 2009, 42 (12), 1910-1921.

(9) Furukawa, H.; Cordova, K. E.; O'Keeffe, M.; Yaghi, O. M., The Chemistry and Applications of Metal-Organic Frameworks. Science 2013, 341 (6149), 974-986.

(10) Shekhah, O.; Chernikova, V.; Belmabkhout, Y.; Eddaoudi, M., Metal-Organic Framework Membranes: From Fabrication to Gas Separation. Crystals 2018, 8 (11), 412-467.

(11) Kreno, L. E.; Leong, K.; Farha, O. K.; Allendorf, M.; Van Duyne, R. P.; Hupp, J. T., Metal-Organic Framework Materials as Chemical Sensors. Chem. Rev. 2012, 112 (2), 1105-1125.

(12) De Luna, P.; Liang, W.; Mallick, A.; Shekhah, O.; García de Arquer, F. P.; Proppe, A. H.; Todorović, P.; Kelley, S. O.; Sargent, E. H.; Eddaoudi, M., Metal-Organic Framework Thin Films on HighCurvature Nanostructures Toward Tandem Electrocatalysis. ACS Appl. Mater. Interfaces 2018, 10 (37), 31225-31232.

(13) Horcajada, P.; Chalati, T.; Serre, C.; Gillet, B.; Sebrie, C.; Baati, T.; Eubank, J. F.; Heurtaux, D.; Clayette, P.; Kreuz, C.; Chang, J. S.; Hwang, Y. K.; Marsaud, V.; Bories, P. N.; Cynober, L.; Gil, S.; Ferey, G.; Couvreur, P.; Gref, R., Porous metal-organic-framework nanoscale carriers as a potential platform for drug delivery and imaging. Nat. Mater. 2010, 9 (2), 172-178.

(14) So, M. C.; Wiederrecht, G. P.; Mondloch, J. E.; Hupp, J. T.; Farha, O. K., Metal-organic framework materials for light-harvesting and energy transfer. Chem. Commun. 2015, 51 (17), 3501-3510.

(15) Sava, D. F.; Rohwer, L. E. S.; Rodriguez, M. A.; Nenoff, T. M., Intrinsic Broad-Band White-Light Emission by a Tuned, Corrugated Metal-Organic Framework. J. Am. Chem. Soc. 2012, 134 (9), 3983-3986.

(16) Sun, J. K.; Cai, L. X.; Chen, Y. J.; Li, Z. H.; Zhang, J., Reversible luminescence switch in a photochromic metal-organic framework. Chem. Commun. 2011, 47 (24), 6870-6872.
(17) Kent, C. A.; Mehl, B. P.; Ma, L. Q.; Papanikolas, J. M.; Meyer, T. J.; Lin, W. B., Energy Transfer Dynamics in Metal-Organic Frameworks. J. Am. Chem. Soc. 2010, 132 (37), 12767-12769.

(18) Sun, L. B.; Xing, H. Z.; Liang, Z. Q.; Yu, J. H.; Xu, R. R., A 4+4 strategy for synthesis of zeolitic metal-organic frameworks: an indium-MOF with SOD topology as a light-harvesting antenna. Chem. Commun. 2013, 49 (95), 11155-11157.

(19) Jin, S. Y.; Son, H. J.; Farha, O. K.; Wiederrecht, G. P.; Hupp, J. T., Energy Transfer from Quantum Dots to Metal-Organic Frameworks for Enhanced Light Harvesting. J. Am. Chem. Soc. 2013, 135 (3), 955-958.

(20)Son, H. J.; Jin, S. Y.; Patwardhan, S.; Wezenberg, S. J.; Jeong, N. C.; So, M.; Wilmer, C. E.; Sarjeant, A. A.; Schatz, G. C.; Snurr, R. Q.; Farha, O. K.; Wiederrecht, G. P.; Hupp, J. T., Light-Harvesting and Ultrafast Energy Migration in Porphyrin-Based Metal-Organic Frameworks. J. Am. Chem. Soc. 2013, 135 (2), 862-869.

(21) Lee, C. Y.; Farha, O. K.; Hong, B. J.; Sarjeant, A. A.; Nguyen, S. T.; Hupp, J. T., Light-Harvesting Metal-Organic Frameworks (MOFs): Efficient Strut-to-Strut Energy Transfer in Bodipy and Porphyrin-Based MOFs. J. Am. Chem. Soc. 2011, 133 (40), 1585815861.

(22)Williams, D. E.; Rietman, J. A.; Maier, J. M.; Tan, R.; Greytak, A. B.; Smith, M. D.; Krause, J. A.; Shustova, N. B., Energy Transfer on Demand: Photoswitch-Directed Behavior of Metal-Porphyrin Frameworks. J. Am. Chem. Soc. 2014, 136 (34), 11886-11889.

(23) Park, J.; Feng, D. W.; Yuan, S.; Zhou, H. C., Photochromic MetalOrganic Frameworks: Reversible Control of Singlet Oxygen Generation. Angew. Chem. Int. Edit. 2015, 54 (2), 430-435.

(24)Park, J.; Jiang, Q.; Feng, D. W.; Zhou, H. C., Controlled Generation of Singlet Oxygen in Living Cells with Tunable Ratios of the Photochromic Switch in Metal-Organic Frameworks. Angew. Chem. Int. Edit. 2016, 55 (25), 7188-7193.

(25) Mallick, A.; El-Zohry, A. M.; Shekhah, O.; Yin, J.; Jia, J. T.; Aggarwal, H.; Emwas, A. H.; Mohammed, O. F.; Eddaoudi, M., Unprecedented Ultralow Detection Limit of Amines using a Thiadiazole-Functionalized $\mathrm{Zr}(\mathrm{IV})$-Based Metal-Organic Framework. J. Am. Chem. Soc. 2019, 141 (18), 7245-7249.

(26)Sk, M.; Biswas, S., A thiadiazole-functionalized $\mathrm{Zr}$ (iv)-based metal-organic framework as a highly fluorescent probe for the selective detection of picric acid. CrystEngComm 2016, 18 (17), 3104-3113.

(27)Sahoo, H., Förster resonance energy transfer - A spectroscopic nanoruler: Principle and applications. J. Photochem. Photobiol., C 2011, 12 (1), 20-30.

(28)Dolgopolova, E. A.; Rice, A. M.; Martin, C. R.; Shustova, N. B., Photochemistry and photophysics of MOFs: steps towards MOFbased sensing enhancements. Chem. Soc. Rev. 2018, 47 (13), 47104728.

(29)Beljonne, D.; Curutchet, C.; Scholes, G. D.; Silbey, R. J., Beyond Förster Resonance Energy Transfer in Biological and Nanoscale Systems. J. Phys. Chem. B 2009, 113 (19), 6583-6599.

(30)Chen, C.-X.; Wei, Z.-W.; Fan, Y.-N.; Su, P.-Y.; Ai, Y.-Y.; Qiu, Q.-F.; Wu, K.; Yin, S.-Y.; Pan, M.; Su, C.-Y., Visualization of Anisotropic and Stepwise Piezofluorochromism in an MOF Single Crystal. Chem 2018, 4 (11), 2658-2669. 


\section{TOC Graphic}

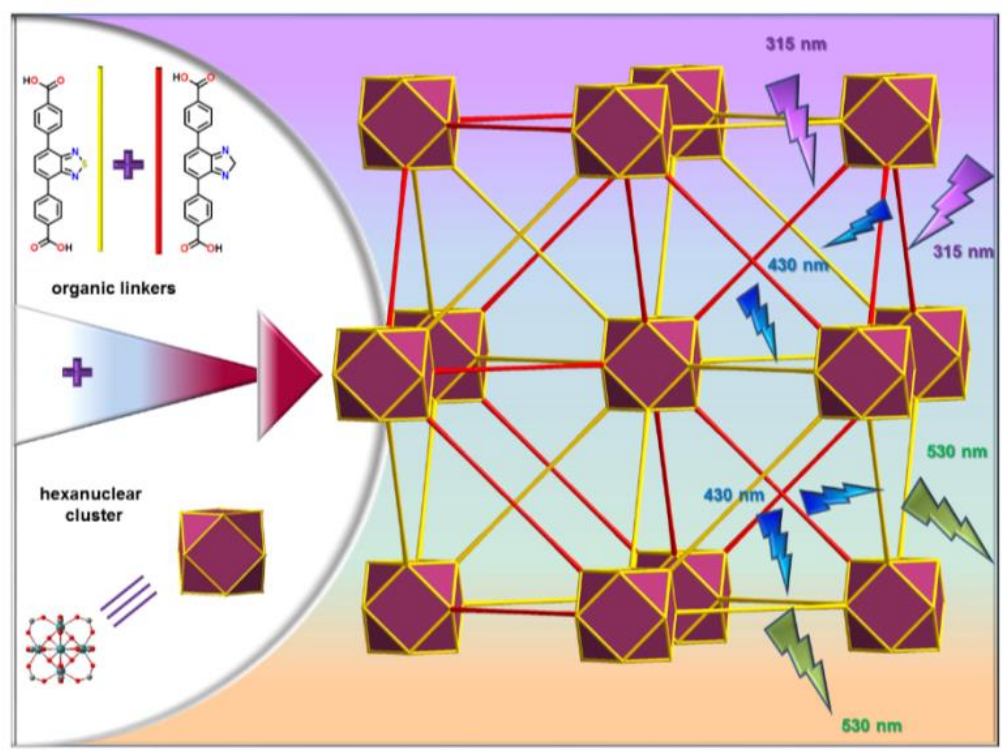

